\title{
Ultrasound elastography: a novel tool for the differential diagnosis of pleural effusion
}

\author{
Bin Jiang ${ }^{1}$, Xue-lian $\mathrm{Li}^{2}$, Yan Yin ${ }^{3}$, Qin Zhang ${ }^{3}$, Tong Zang ${ }^{4}$, Wang-shu Song ${ }^{1}$, \\ Xue-mei Wang ${ }^{1}$, Jian Kang ${ }^{3}$, Felix J.F. Herth ${ }^{5}$ and Gang Hou $\mathbb{B}^{3}$ \\ Affiliations: ${ }^{1}$ Dept of Ultrasound, The First Hospital of China Medical University, Shenyang, China. ${ }^{2}$ Dept of \\ Epidemiology, School of Public Health, China Medical University, Shenyang, China. ${ }^{3}$ Dept of Respiratory and \\ Critical Care Medicine, The First Hospital of China Medical University, Shenyang, China. ${ }^{4}$ Dept of Ultrasound, \\ The Central Hospital of Shenyang Medical College, Shenyang, China. ${ }^{5}$ Dept of Pneumology and Critical Care \\ Medicine, Thoraxklinik University of Heidelberg, Heidelberg, Germany.
}

Correspondence: Gang Hou, Dept of Respiratory and Critical Care Medicine, First Hospital of China Medical University, No. 155 Nanjing Street, Shenyang, China. E-mail: hougangcmul163.com

@ERSpublications

Pleural ultrasound elastography is a better technique than traditional thoracic ultrasound for diagnosing malignant pleural effusion http://bit.ly/2VX4Qns

Cite this article as: Jiang B, Li X-lian, Yin Y, et al. Ultrasound elastography: a novel tool for the differential diagnosis of pleural effusion. Eur Respir J 2019; 54: 1802018 [https://doi.org/10.1183/13993003.020182018].

\section{ABSTRACT}

Introduction: Traditional thoracic ultrasound (TUS) is often the initial tool used to help diagnose malignant pleural effusion (MPE). Ultrasound elastography, a relatively new technique, has been used to differentiate malignant disease from benign disease by evaluating tissue "stiffness". However, no studies evaluating the efficacy of ultrasound elastography for diagnosing MPE are available. We assessed the value of ultrasound elsatography for diagnosing MPE prospectively.

Methods: All 244 enrolled patients were divided into a development set and a validation set in chronological order. The cut-off elasticity index was established using a receiver operating characteristic curve constructed from the continuous data of the patients in the development set. The diagnostic performance of ultrasound elastography was compared with that of TUS in the validation set.

Results: In the development set, the mean elasticity index $(47.25 \mathrm{kPa})$ was the optimal cut-off. In the validation set, pleural ultrasound elastography had a sensitivity of $83.64 \%$, a specificity of $90.67 \%$, a positive predictive value of $86.79 \%$, a negative predictive value of $88.31 \%$, a positive likelihood ratio of 8.96 and a negative likelihood ratio of 0.18 for diagnosing MPE. The sensitivity of ultrasound elastography was significantly higher $(\mathrm{p}=0.006)$ than that of TUS $(60 \%)$.

Conclusion: Pleural ultrasound elastography is a better technique than TUS for differentiating MPE from benign pleural disease.

\footnotetext{
This article has supplementary material available from erj.ersjournals.com
}

Received: 23 Oct 2018 | Accepted after revision: 22 May 2019

Copyright OERS 2019 


\section{Introduction}

Pleural effusion is an extremely common problem; however, the diagnosis of pleural effusion remains challenging due to its diverse aetiologies. Malignant pleural effusion (MPE) is one of the leading causes of unilateral pleural effusion, and many clinical guidelines have recommended diagnostic strategies for MPE $[1,2]$. Radiographic techniques, such as contrast-enhanced computed tomography and thoracic ultrasound (TUS), have been shown to be valuable in the diagnosis of MPE. Ultrasound imaging is convenient, free of radiation and can be used to diagnose pleural disease, guide closed pleural biopsy and assess the characteristics of pleural effusion [1]. Ultrasound elastography is a recently developed, novel ultrasound technology that can be used to quantitatively assess tissue stiffness by measuring the degree of distortion under the application of an external force (shear waves). Since tumour tissue is stiffer than normal tissue, ultrasound elastography has been used to evaluate tissue stiffness and is a very valuable imaging method for differentiating malignant from benign disease, such as breast [3], thyroid [4] and liver diseases [5, 6]. However, to the best of our knowledge, no published studies have assessed the diagnostic value of ultrasound elastography in MPE. We conducted this prospective study to determine the cut-off elasticity index for the diagnosis of MPE and to assess the diagnostic accuracy of ultrasound elastography compared with TUS for differentiating MPE from benign pleural effusion.

\section{Methods}

\section{Study design}

The study was conducted prospectively at the First Hospital of China Medical University (Shenyang, China) between October 2012 and October 2017. Patients with unilateral or bilateral pleural effusion were enrolled in this study. The inclusion criteria were as follows. 1) Patients with pleural effusion, as demonstrated by chest radiography; 2) undiagnosed effusions (malignant or otherwise) at the first patient encounter or presentation; 3) patients who did not undergo thoracentesis, drainage and analysis of pleural effusions before enrolment; and 4) patients who, in clinical practice, would have undergone further examination to establish the cause of pleural effusion. The exclusion criterion was inability or refusal to undergo further examination to establish the cause of pleural effusion. All enrolled patients were divided into a development set and a validation set based on the chronological order in which they presented to the hospital (figure 1). The primary outcome was the capacity of ultrasound elastography to identify MPE compared with traditional TUS. The secondary outcome was the influence of different aetiologies on the diagnostic performance of ultrasound elastography. This study was approved by the institutional ethical review board of the First Hospital of China Medical University (reference number: 201-9126-2), and written informed consent was obtained from all patients.

To obtain the desired level of statistical power for evaluating the accuracy of the diagnostic test, the minimal sample size required was calculated based on the estimated sensitivity and specificity [7]:

$$
N_{S e}=\frac{Z \frac{\alpha^{2}}{2} \times \widehat{S e} \times(1-\widehat{S e})}{d^{2} \times \text { Prev }} ; \quad N_{S p}=\frac{Z \frac{\alpha^{2}}{2} \times \widehat{S p} \times(1-\widehat{S p})}{d^{2} \times(1-\text { Prev })} ;
$$

We estimated the sensitivity (Se) and specificity (Sp) for the mean elasticity index to diagnose MPE as $85 \%$ and $85 \%$, respectively, according to our preliminary pilot study of a small sample of 20 cases; we estimated the prevalence of MPE in patients with pleural effusion as $43 \%$ according to data from our centre in 2012. For a maximum marginal error of the estimate not exceeding $10 \%$ with a $95 \%$ confidence level, the total required sample size was 114 patients. All 114 patients needed were enrolled in the development set to measure the accuracy of interest (the sensitivity/specificity and the area under the receiver operating characteristic (ROC) curve (AUC)), and another 130 patients were enrolled in the validation set.

\section{Transthoracic ultrasound and ultrasound elastography}

Before pleural ultrasound, a respiratory expert $(\mathrm{GH})$ reviewed each patient's most recent chest radiographs to determine which side of the thorax to assess via ultrasound. In patients with bilateral pleural effusion, the side with the greater amount of effusion was assessed, since this was usually considered the clinically relevant side. Two operators with different seniority levels (the senior operator was a radiologist with $>8$ years of experience, while the junior operator was a radiologist with 3 years of experience) who were blinded to the patients' clinical history and thoracic imaging (computed tomography/magnetic resonance imaging) data performed all ultrasound examinations separately.

Greyscale ultrasound was performed with an Aixplorer ultrasound scanning system (SuperSonic Imagine, Aix-en-Provence, France) with a 4 - to $15-\mathrm{MHz}$ linear-array transducer, as described in previous studies 


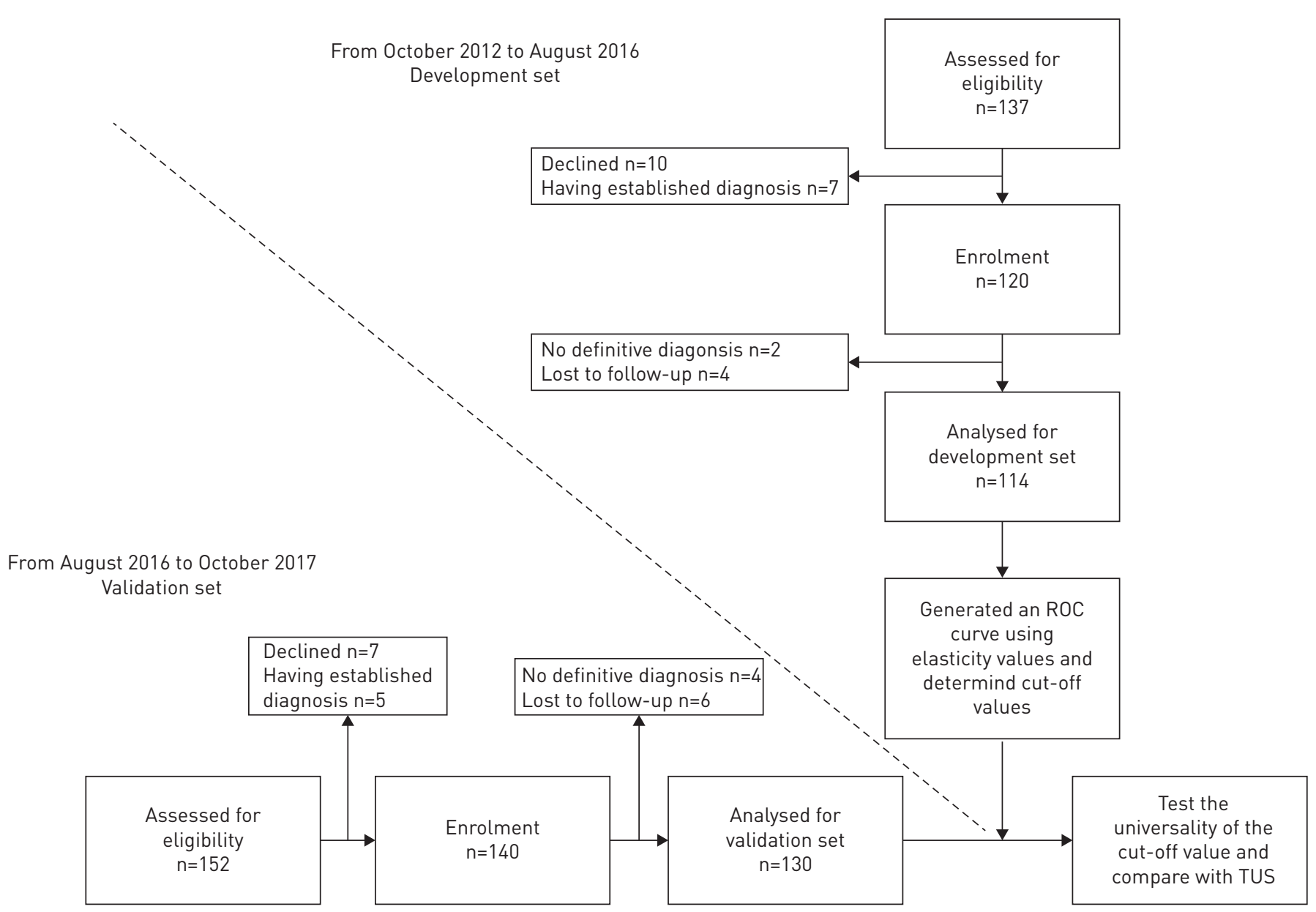

FIGURE 1 Flow chart of patient enrolment. ROC: receiver operating characteristic; TUS: traditional thoracic ultrasound.

[8-10]. The ultrasound imaging features of pleural nodules and focal pleural thickening are shown in figure $2 \mathrm{a}, \mathrm{b}$.

Measurements of pleural stiffness were performed using shear wave elastography (SWE) $[11,12]$ (figure 2c). Using the device's software (OsiriX, version 6.0; Pixmeo, Berne, Switzerland), a circular region of interest was placed inside the pleural elastogram, and the diameter of the circle was increased as much as possible to between 1 and $8 \mathrm{~mm}$, taking care not to surpass the limits of the analysed pleurae. The default setting for the pleural 2D-SWE scale was used (range $0-100 \mathrm{kPa}$ ). A summary of the quantitative stiffness data was automatically displayed. The following parameters for the elasticity index, expressed in $\mathrm{kPa}$, were provided by the system: the mean elasticity index (SWEmean), the maximum elasticity index (SWEmax), the minimum elasticity index, and the elasticity index standard deviation [13]. Precise details of the TUS and ultrasound elastography technique are provided in the supplementary material.

Criteria for malignant pleural disease on TUS

The operators separately recorded an initial diagnosis of malignant or benign pleural disease pro forma on the basis of anonymised TUS data. If patients had any one of the following criteria upon TUS examination, then a TUS-based diagnosis of malignant disease was recorded: 1) diaphragmatic or parietal pleural nodule(s); 2) pleural thickening $>1 \mathrm{~cm}$; or 3) hepatic metastasis [14]. If patients had none of those three criteria, benign pleural effusion based on TUS would be recorded.

\section{Pleural effusion types and the criteria for definitive diagnosis}

The diagnosis of malignant pleural lesions was confirmed by the histocytological examination of biopsy samples obtained by semirigid thoracoscopy or closed pleural biopsy or by the cytopathological examination of pleural effusion samples using a liquid-based thin-layer cytopathology technique [1]. When disseminated malignancy was present with no alternative explanation for exudative effusion, probable MPE was diagnosed. 


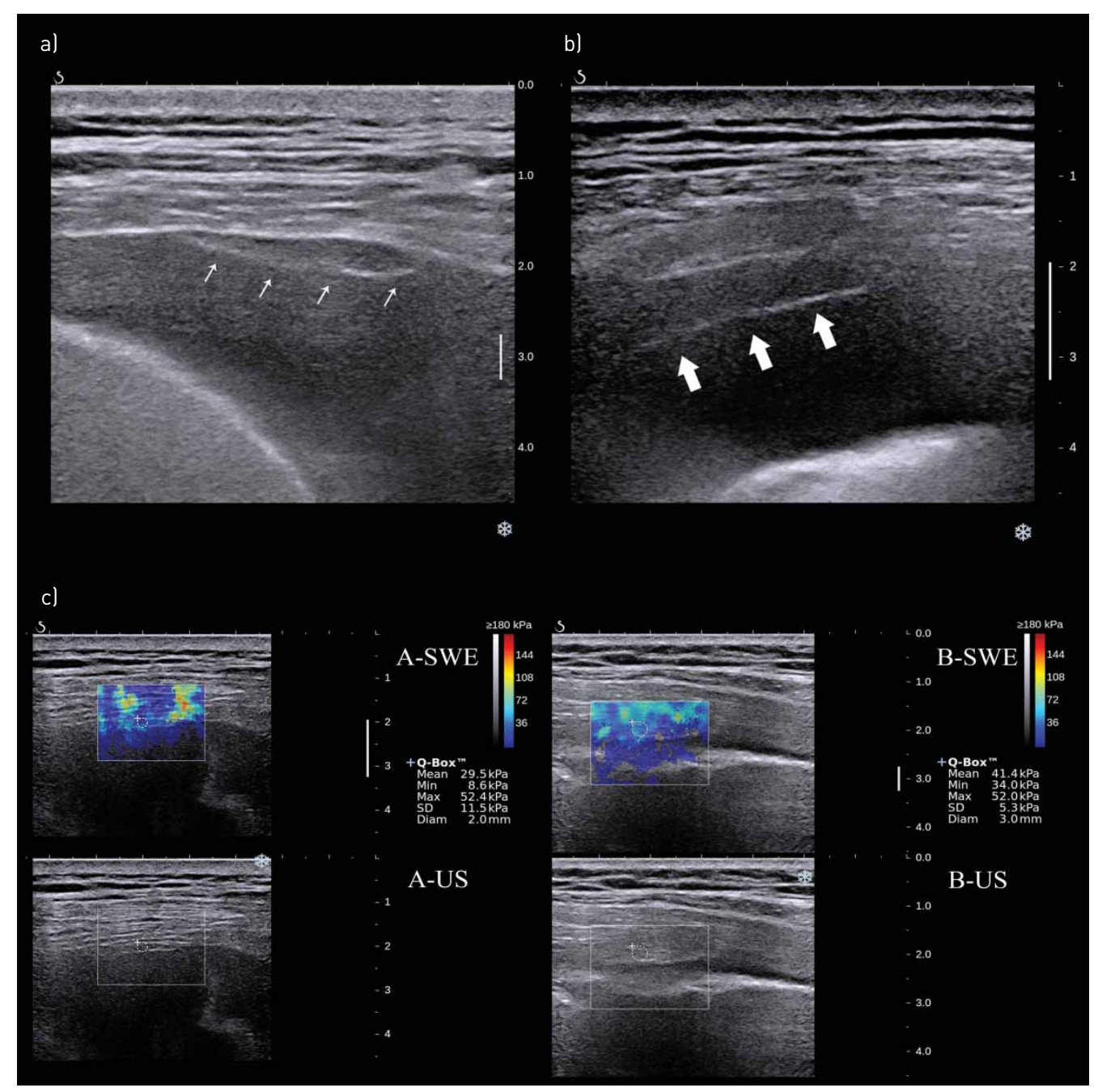

FIGURE 2 Ultrasound features of pleural nodules and focal pleural thickening and measurements of pleural stiffness by shear wave elastography. al Pleural nodules appeared as hypoechoic nodular lesions with defined margins located in the parietal or visceral pleura, while b) focal pleural thickening was identified as an echogenic area of increased thickness in the parietal pleura with poorly defined margins. c) Images of pleural thickening or nodules are displayed together with the greyscale ultrasound images. After placing a box (frame) over the pleura, a coloured image appeared, revealing blue and red areas on an elastogram. Dark blue areas correspond to soft tissues, whereas red areas correspond to stiff tissues.

All definitive diagnoses of benign pleural effusion were determined based on comprehensive clinical data, including imaging, laboratory results and histocytological manifestations of disease. No patients with benign disease had any evidence of malignancy during the follow-up period of $>12$ months [15]. Benign pleural effusion was definitively diagnosed using pathogenic microorganism isolation methods, such as those used for diagnosing tuberculosis (TB) and empyema. Tubercular pleurisy was diagnosed based on a positive mycobacterial stain/culture of pleural fluid or pleural tissue or the presence of caseous granuloma on pleural biopsy. Empyema was diagnosed if thoracentesis yielded frank pus and/or bacteria were identified by Gram staining or the culture of pleural effusion samples [16]. Parapneumonic effusion was considered exudative effusion that occurred in the presence of clinical evidence of pneumonia and the exclusion of other suspicious diagnoses. The classification of the effusion as exudate or transudate was based on Light et al.'s criteria [17].

The methods used for determining the final diagnoses are shown in table 1. In 52 (48.15\%) out of 108 patients, the definitive diagnosis of MPE was based on the pleural histocytological results. In addition, 56 (51.85\%) out of 108 patients were diagnosed with probable MPE based on clinical criteria.

\section{Statistical analysis}

All data were collected and analysed using SPSS 17.0 (SPSS for Windows, version 17.0; SPSS, Chicago, IL, USA). For the ultrasound elastography and TUS data, we used paired t-tests and $\kappa$ coefficients to determine 
TABLE 1 Methods used for the final diagnosis of malignant pleural effusions

$\begin{array}{lr}\text { Definitive diagnosis of malignant disease } & 52 \\ \text { Thoracoscopic biopsy } & 39 \\ \text { Percutaneous pleural biopsy } & 4 \\ \text { Pleural fluid cytology } & 9 \\ \text { Probable diagnosis }{ }^{\#} \text { of malignant disease } & 56 \\ \text { Histocytological evidence of primary tumour and clinical follow-up (>6 months) } & 49 \\ \text { Clinical follow-up (>6 months) and repeated radiography } & 7\end{array}$

Data are presented as $n$. ${ }^{\#}$ : there were no aetiologies other than cancer that could explain these cases of pleural effusion according to clinical criteria.

the interobserver agreement between the two operators with different seniority levels, and the findings of the senior radiologist (BJ) were used for all subsequent analyses. Statistical analysis of the differences in diagnoses was performed using the exact binomial test. The elasticity indices of the development set, as continuous data, were used to construct ROC curves. The AUC was used as a performance measure. We attempted to establish the optimal cut-off values for SWEmean and SWEmax using YoudEN's index [18]. In the validation set, we tested the universality of the cut-off values for differentiating MPE from benign pleural effusion. The diagnostic efficacy (sensitivity, specificity, positive predictive value (PPV), negative predictive value (NPV)), positive likelihood ratio (PLR), negative likelihood ratio (NLR) and Youden's index) of ultrasound elastography at fixed cut-off values was compared with that of traditional TUS. The 95\% confidence interval of sensitivity, specificity, PPV, NPV, PLR and NLR was calculated using an online clinical calculator (http://vassarstats.net/clin1.html). Statistical significance was set at $\mathrm{p}<0.05$.

\section{Results}

Baseline patient characteristics

A total of 260 consecutive patients with pleural effusion were recruited for this study. Of these patients, 244 were enrolled in the final analysis. The baseline characteristics of these patients are shown in table 2.

\section{Interobserver agreement of ultrasound elastography and TUS between two operators with different seniority levels}

Good interobserver agreement was observed between the two operators when the SWEmean $(\mathrm{p}=0.976)$ and SWEmax $(p=0.581)$ were analysed using paired t-tests. In addition, similar agreement was observed between the two operators in terms of the overall diagnosis of malignant or benign pleural effusion based on TUS (the sensitivity of the senior operator was $58.33 \%$ and that of the junior operator was $50.00 \%$; $\kappa$ coefficient $=0.844, \mathrm{p}<0.001)$. The results of the more experienced operator were used in the subsequent analysis of all results, and the results of the less experienced operator are given in the supplementary material.

\section{Diagnostic performance of TUS}

TUS provided a correct diagnosis for $30(56.60 \%)$ out of 53 patients with malignant disease and 55 $(90.16 \%)$ out of 61 patients with benign disease in the development set, and Youden's index was 46.76 (table 3). In the validation set, the sensitivity of TUS was $60.00 \%$, the specificity was $93.33 \%$ and Youden's index was 53.33 .

\section{Derivation of cut-off elasticity indices based on pleural ultrasound elastography for detecting MPE}

The ROC curves derived from the development set that demonstrate the diagnostic capacity of ultrasound elastography to identify MPE based on the SWEmean and SWEmax are shown in figure 3. The diagnostic evaluation of these cut-off values is presented in table 3. In the development set, the diagnostic performance based on SWEmean $\geqslant 47.25 \mathrm{kPa}$ showed that $88.60 \%$ (101 out of 114) of patients were diagnosed correctly; Youden's index was 77.46. Eight patients with benign pleural effusion were misdiagnosed with MPE (tuberculous pleurisy $\mathrm{n}=6$ and empyema $\mathrm{n}=2$ ), and five MPE patients were missed. The diagnostic performance based on SWEmax $\geqslant 56.9 \mathrm{kPa}$ showed that $84.21 \%$ (96 out of 114 ) of patients were diagnosed correctly; Youden's index was 68.76. 11 patients with benign pleural effusion were misdiagnosed with MPE (tuberculous pleurisy $\mathrm{n}=8$ and empyema $\mathrm{n}=3$ ), and seven MPE patients were missed (supplementary material). 


\begin{tabular}{|c|c|c|c|c|}
\hline & All patients & Development set & Validation set & p-value $\#$ \\
\hline Subjects $\mathrm{n}$ & 244 & 114 & 130 & \\
\hline Age years & $\begin{array}{c}56.15 \pm 14.76 \\
58(16-94)\end{array}$ & $\begin{array}{l}55.93 \pm 15.18 \\
57.5(16-87)\end{array}$ & $\begin{array}{c}56.34 \pm 14.44 \\
58(21-94)\end{array}$ & 0.830 \\
\hline Male/female & $150 / 94$ & $68 / 46$ & $82 / 48$ & 0.583 \\
\hline Diagnosis & & & & 0.512 \\
\hline Malignant & $108(44.26)$ & 53 (46.49) & 55 (42.31) & \\
\hline Benign & $136(55.74)$ & $61(53.51)$ & 75 (57.69) & \\
\hline \multicolumn{5}{|l|}{ Final diagnosis } \\
\hline Malignant & $108(44.26)$ & 53 (46.49) & $55(42.31)$ & \\
\hline Definitive diagnosis & $52(48.15)$ & $33(62.26)$ & 19 (34.55) & \\
\hline Probable diagnosis & 56 (51.85) & $20(37.74)$ & $36(65.45)$ & \\
\hline Benign & $136(55.74)$ & $61(53.51)$ & 75 (57.69) & \\
\hline Pneumonia & $44(18.03)$ & $22(19.30)$ & $22(16.92)$ & \\
\hline Tuberculous pleurisy & 45 (18.44) & 33 (28.95) & $12(9.23)^{\S}$ & \\
\hline Congestive heart failure & $11(4.51)$ & $2(1.75)$ & $9(6.92)$ & \\
\hline Hepatic disease & $22(9.02)$ & $3(2.63)$ & 19 (14.62) & \\
\hline Renal failure & $8(3.28)$ & $0(0)$ & $8(6.15)$ & \\
\hline Other benign disease ${ }^{+}$ & $6(2.46)$ & $1(0.88)$ & 5 (3.85) & \\
\hline
\end{tabular}

Data are presented as $\mathrm{n}$, mean $\pm \mathrm{SD}$, median (range) or $\mathrm{n}(\%)$, unless otherwise stated. " : comparison of the development set with the validation set; " : refers to lung cancer $(n=40)$, mesothelioma ( $n=5)$, thymic tumour $(n=1)$, breast cancer $(n=1)$, gastrointestinal cancer $(n=1)$ and unclassified malignancy $(n=5)$ in the development set, and refers to lung cancer $(n=34)$, mesothelioma $(n=4)$, thymic tumour $(n=3)$, breast cancer $(n=6)$, gastrointestinal cancer $(n=4)$ and unclassified malignancy $(n=4)$ in the validation set; ${ }^{+}$: refers to Sjogren syndrome $(n=1)$ in the development set and systemic sclerosis $(n=1)$, systemic lupus erythematosus $(n=3)$ and Sjogren syndrome $(n=1)$ in the validation set; ${ }^{\S}$ : one patient suffered from tuberculous pleurisy and empyema and was listed only once, under tuberculous pleurisy.

\section{Diagnostic performance of pleural ultrasound elastography}

In the validation set, our data showed a sensitivity of $83.64 \%$ (46 out of 55) and a specificity of $90.67 \%$ (68 out of 75) for detecting MPE with the SWEmean (table 3). A total of 114 (87.69\%) out of 130 patients were correctly diagnosed, and Youden's index was 74.31; seven patients with benign pleural effusion were misdiagnosed with MPE (tuberculous pleurisy $n=5$, spontaneous bacterial pleural effusion transudate $n=1$

\begin{tabular}{|c|c|c|c|c|c|c|c|}
\hline & TUS & SWEmean & Chi-squared $^{\#}$ & p-value ${ }^{\#}$ & SWEmax & Chi-squared ๆ & p-value ${ }^{\text {I }}$ \\
\hline \multicolumn{8}{|l|}{ Development set } \\
\hline Yield \% & 74.56 & 88.60 & & & 84.21 & & \\
\hline PPV $\%(95 \%$ CI $)$ & $83.33(66.53-93.04)$ & $85.71(73.22-93.20)$ & 15.725 & $<0.001$ & $80.70(67.68-89.53)$ & 11.902 & 0.001 \\
\hline NPV $\%(95 \% \mathrm{Cl})$ & $70.51(58.96-80.03)$ & 91.38 (80.28-96.78) & & & $87.72(75.71-94.51)$ & & \\
\hline $\operatorname{PLR}(95 \% \mathrm{CI})$ & $5.75(2.60-12.75)$ & $6.91(3.60-13.25)$ & & & $4.81(2.79-8.30)$ & & \\
\hline NLR $(95 \% \mathrm{CI})$ & $0.48(0.35-0.66)$ & $0.11(0.05-0.25)$ & & & $0.16(0.08-0.32)$ & & \\
\hline \multicolumn{8}{|l|}{ Validation set } \\
\hline Yield \% & 79.23 & 87.69 & & & 84.62 & & \\
\hline NLR $(95 \% \mathrm{CI})$ & $0.43(0.31-0.59)$ & $0.18(0.10-0.33)$ & & & $0.26(0.16-0.42)$ & & \\
\hline
\end{tabular}

SWE: shear wave elastography; SWEmean: mean SWE elasticity index; SWEmax: maximum elasticity index; PPV: positive predictive value; NPV: negative predictive value; PLR: positive likelihood ratio; NLR: negative likelihood ratio. "\#: comparison of sensitivity between traditional TUS and SWEmean; ": comparison of sensitivity between traditional TUS and SWEmax. 
FIGURE 3 Receiver operating characteristic curve analysis of the mean and maximal elasticity indexes of pleural ultrasound elastography for diagnosing malignant pleural effusion in the development set. Diagonal segments are produced by ties. SWE: shear wave elastography; SWEmean: mean SWE elasticity index; SWEmax: maximal SWE elasticity index.

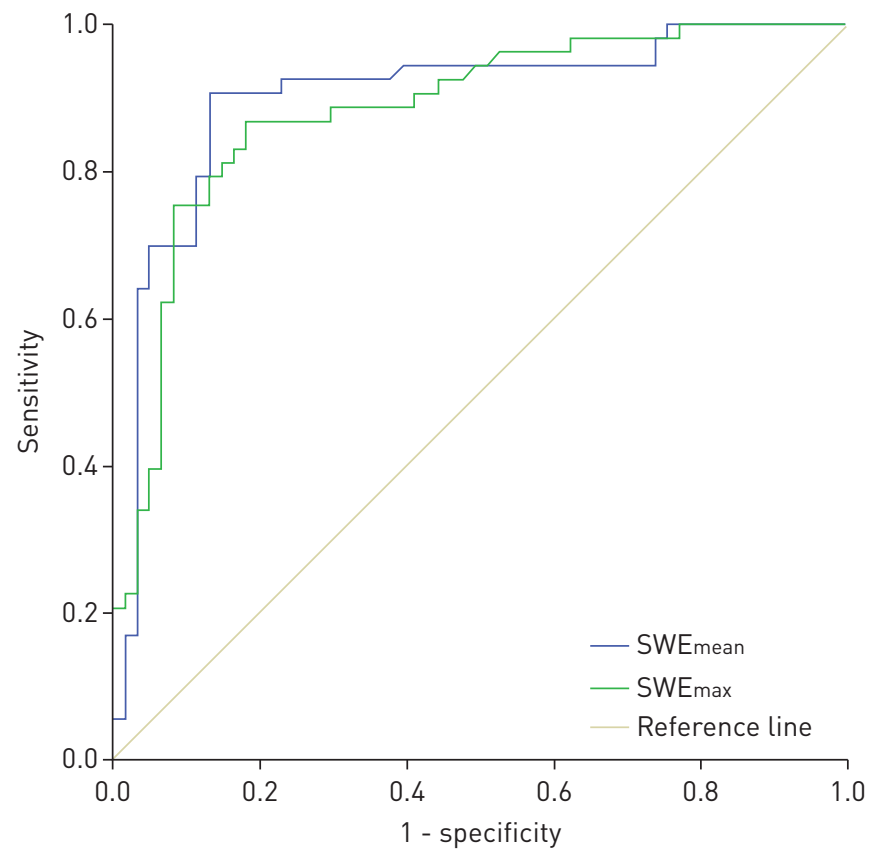

and empyema $\mathrm{n}=1$ ), and nine MPE patients were missed. In terms of SWEmax, the analysis showed a sensitivity of $76.36 \%$ (42 out of 55) and a specificity of $90.67 \%$ (68 out of 75 ). In all, 110 (84.62\%) out of 130 patients were correctly diagnosed, and Youden's index was 67.03; seven patients with benign pleural effusion were misdiagnosed with MPE (tuberculous pleurisy $n=4$, pleural transudate $n=1$, pneumonia $n=1$ and empyema $\mathrm{n}=1$ ), and 13 MPE patients were missed. The diagnostic yield of both SWEmean and SWEmax was slightly lower in the validation set than in the development set, but the differences were not significant ( $\mathrm{p}=0.190$ and 0.053 , respectively).

Comparison of diagnostic sensitivity and agreement between different techniques

In the validation set, the sensitivity of SWEmean was significantly higher than that of TUS (Chi-squared=7.591, p=0.006) (table 3). SWEmean and SWEmax both showed low agreement with TUS ( $\kappa$ coefficient $=0.350$ and 0.263 , respectively). There were complementary effects of TUS and ultrasound elastography on the diagnosis of MPE (table 4). The combination of TUS and ultrasound elastography (either SWEmean or SWEmax) for diagnosing MPE, where the test result is considered positive if either measure is positive, had 100\% (95\% CI 91.87-100\%) sensitivity, 86.67\% (95\% CI 76.39-93.08\%) specificity,

\begin{tabular}{|c|c|c|c|c|}
\hline \multirow{5}{*}{$\begin{array}{l}\text { Development set } \\
\text { SWEmean/SWEmax }\end{array}$} & \multicolumn{4}{|c|}{ TUS } \\
\hline & & + & - & Total \\
\hline & + & $29 / 28$ & $19 / 18$ & $48 / 46$ \\
\hline & - & $1 / 2$ & $4 / 5$ & $5 / 7$ \\
\hline & Total & 30 & 23 & 53 \\
\hline \multirow{5}{*}{$\begin{array}{l}\text { Validation set } \\
\text { SWEmean/SWEmax }\end{array}$} & \multicolumn{4}{|c|}{ TUS } \\
\hline & & + & - & Total \\
\hline & + & $24 / 20$ & $22 / 22$ & $46 / 42$ \\
\hline & - & $9 / 13$ & $0 / 0$ & $9 / 13$ \\
\hline & Total & 33 & 22 & 55 \\
\hline \multirow{5}{*}{$\begin{array}{l}\text { All patients } \\
\text { SWEmean/SWEmax }\end{array}$} & \multicolumn{4}{|c|}{ TUS } \\
\hline & & + & - & Total \\
\hline & + & $53 / 48$ & $41 / 40$ & $94 / 88$ \\
\hline & - & $10 / 15$ & $4 / 5$ & $14 / 20$ \\
\hline & Total & 63 & 45 & 108 \\
\hline
\end{tabular}

Data are presented as n. SWE: shear wave elastography; SWEmean: mean SWE elasticity index; SWEmax: maximum SWE elasticity index. 
FIGURE 4 Receiver operating characteristic curve analysis of the mean and maximal elasticity indexes of pleural ultrasound elastography for diagnosing malignant pleural effusion in the validation set. Diagonal segments are produced by ties. SWE: shear wave elastography; SWEmean: mean SWE elasticity index; SWEmax: maximal SWE elasticity index.

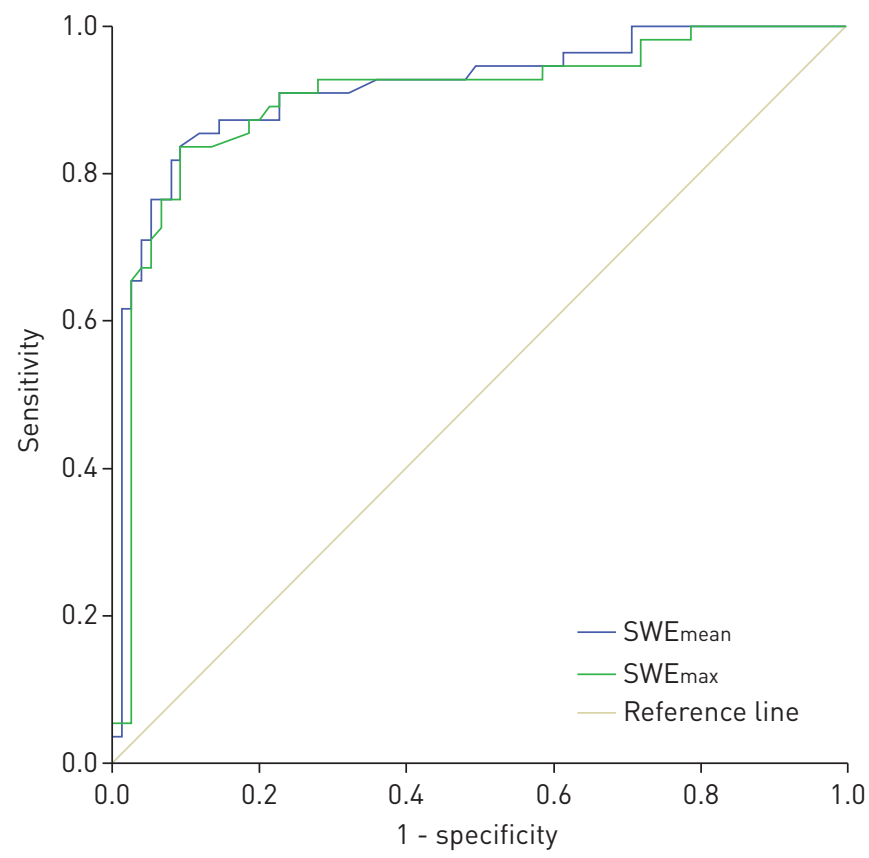

a PLR of 7.50 (95\% CI 4.21-13.36) and an NLR of 0. The AUC of SWEmean and SWEmax for detecting MPE in the validation set was 0.915 (95\% CI $0.862-0.968$ ) and 0.901 (95\% CI 0.841-0.960), respectively (figure 4). The diagnostic performance of ultrasound elastography for detecting MPE based on SWEmean compared with SWEmax showed no significant difference (Chi-squared=0.258, $\mathrm{p}=0.611$ ) and a high level of agreement $(\kappa$ coefficient $=0.903$ ). Additionally, among the 108 cases of MPE among all the enrolled patients, pleural ultrasound elastography (SWEmean) detected $91.11 \%$ of the cases (41 out of 45) missed by TUS.

\section{Comparison of the diagnostic sensitivity of TUS and ultrasound elastography in different} subgroups

The diagnostic sensitivity of TUS, SWEmean and SWEmax was compared according to disease type (table 5). The sensitivity of both SWEmean and SWEmax was significantly higher than the sensitivity of TUS in patients with metastatic cancer $(\mathrm{p}<0.001$ for both measures), whereas SWEmean and SWEmax both showed a significantly lower sensitivity than TUS for tuberculous pleurisy ( $\mathrm{p}=0.027$ and $\mathrm{p}=0.015$, respectively).

TABLE 5 Diagnostic sensitivity of traditional thoracic ultrasound (TUS) and the cut-off mean and maximal elasticity indices of ultrasound elastography in different subgroups

\begin{tabular}{|c|c|c|c|c|c|}
\hline & \multirow{2}{*}{$\begin{array}{c}\text { TUS } \\
\text { Sensitivity }\end{array}$} & \multicolumn{2}{|c|}{ SWEmean } & \multicolumn{2}{|c|}{ SWEmax } \\
\hline & & Sensitivity & p-value ${ }^{\#}$ & Sensitivity & p-value \\
\hline \multicolumn{6}{|l|}{ Malignant disease } \\
\hline Mesothelioma & $88.89(8 / 9)$ & 100 (9/9) & 1 & $100(9 / 9)$ & 1 \\
\hline Metastatic cancer & 55.56 (55/99) & 85.86 (85/99) & $<0.001$ & 79.80 (79/99) & $<0.001$ \\
\hline Definitive MPE & $55.77(29 / 52)$ & $92.31(48 / 52)$ & $<0.001$ & $82.69(43 / 52)$ & 0.003 \\
\hline \multicolumn{6}{|l|}{ Benign disease } \\
\hline Tuberculous pleurisy $^{+}$ & $91.11(41 / 45)$ & 73.33 (33/45) & 0.027 & $71.11(32 / 45)$ & 0.015 \\
\hline Empyema & $66.67(4 / 6)$ & $50(3 / 6)$ & 1 & $33.33(2 / 6)$ & 0.567 \\
\hline Transudate & $93.62(44 / 47)$ & $97.87(46 / 47)$ & 0.617 & $97.87(46 / 47)$ & 0.617 \\
\hline Parapneumonic effusion $\S$ & $94.87(37 / 39)$ & $100(39 / 39)$ & 0.494 & $97.44(38 / 39)$ & 1 \\
\hline
\end{tabular}

Data are presented as \% $(\mathrm{n} / \mathrm{N})$, unless otherwise stated. SWE: shear wave elastography; SWEmean: mean SWE elasticity index; SWEmax: maximum SWE elasticity index; MPE: malignant pleural effusion. ${ }^{\#}$ : comparison of sensitivity between TUS and SWEmean; ${ }^{~}$ : comparison of sensitivity between TUS and SWEmax; ${ }^{+}$: one patient had both tuberculosis pleurisy and empyema; ${ }^{\circledR}$ : excluding empyema. 
There was no significant difference between definitive MPE and probable MPE diagnosed by TUS, SWEmean and SWEmax $(p=0.525, p=0.170$ and $p=0.906$, respectively).

\section{Discussion}

TUS is a cost-effective, non-invasive technique that is helpful for evaluating undiagnosed pleural exudate [1]. Our study demonstrates that ultrasound elastography had a better sensitivity for detecting MPE than TUS when the SWEmean was set at $47.25 \mathrm{kPa}$. Ultrasound elastography is a novel ultrasound technique that is widely accepted for evaluating diseases of the liver, breast and thyroid gland; ultrasound elastography is rapid, relatively inexpensive and free of radiation and can be used for the diagnosis of MPE based on the assessment of the physical properties of pleura. A diagnosis of MPE made by TUS is based on pleural morphological criteria, such as pleural thickening and the presence of nodules, but many patients do not exhibit these specific features. Pleural ultrasound elastography overcomes for this limitation because it is used to diagnose MPE based on pleural stiffness. In our study, pleural ultrasound elastography (SWEmean) detected $91.11 \%$ of patients with MPE missed by TUS.

Only two previous studies have evaluated the efficacy of TUS for diagnosing suspected MPE; these studies were conducted in the UK and Portugal and reported a sensitivity of $79 \%$ and $80.3 \%$, respectively, and a specificity of $100 \%$ and $83.6 \%$, respectively [14, 19]. Our results demonstrate that SWEmean had a sensitivity (83.64\%) similar to that of TUS in previous studies $[14,19]$. Considering the low diagnostic agreement $(\kappa$ coefficient $=0.350$ ) between SWEmean and TUS, ultrasound elastography and TUS could be complementary for diagnosing MPE.

Our data revealed a specificity of $90.67 \%$ for ultrasound elastography, which was slightly lower than that of TUS in our study (93.33\%) and in the previous study conducted in the UK (100\%) [14]. However, the two aforementioned studies [14,19] only focused on European populations. This difference may be explained by the difference in aetiology spectra of pleural effusion between the European and Chinese population. The relatively high ratio of tuberculous pleurisy in our study may result in the decrease of specificity for diagnosing MPE by ultrasound elastography.

In mesothelioma cases, ultrasound elastography (nine out of nine patients) performed as well as TUS (eight out of nine patients) $(\mathrm{p}=1.000)$. Marked heterogeneity exists between countries in the incidence of malignant mesothelioma. The crude incidence of mesothelioma in some European countries ranges from 10 to 30 cases per million [20], with the highest annual incidence (29 cases per million in 2009) in the UK [21]; this is in sharp contrast to the low incidence of mesothelioma in Asian countries (2-9 cases per million) [20]. In particular, QURESHi et al. [14] performed a study in a tertiary pleural centre, and their cohort had a high proportion of mesothelioma cases (14 out of 33).

Tuberculous pleurisy is the second most common extrapulmonary manifestation of TB [22], and its most common sequela is residual pleural thickening. Pleural thickening $(>2 \mathrm{~mm}$ ) has been reported to occur in $\sim 50 \%$ of cases [23]. False-positive results due to tuberculous pleurisy are a common problem in ultrasound elastography and TUS examinations. In our study, there were four false-positive results based on TUS and 12 false-positive results based on SWEmean in 45 patients with tuberculous pleurisy, which is consistent with this phenomenon. Only two patients with tuberculous pleurisy were reported out of a total of 52 patients in the study by QURESHI et al. [14], which is consistent with the low incidence of TB in the UK. However, in our study, 45 out of a total of 244 patients had tuberculous pleurisy, which is consistent with the high burden of TB in China, and may have reduced the specificity of ultrasound elastography in this study. In low-income developing countries, TB remains an important cause of disease burden and may interfere with the use of TUS and ultrasound elastography for detecting MPE.

Additionally, in a subgroup of patients with empyema, TUS and ultrasound elastography (SWEmean) detected only three and two cases, respectively, out of the five cases of empyema (non-tubercular pleuritis). The low diagnostic yield of ultrasound elastography and TUS in patients with empyema may be due to severe irritation of the pleura leading to thickening [24] and changes in elasticity [25].

TUS and ultrasound elastography (SWEmean) correctly detected 93.62\% (44 out of 47) and 97.87\% (46 out of 47) of patients with benign effusion in the transudate subgroup, respectively. Such a high diagnostic yield is important for elderly patients with pleural effusion in whom the differential diagnoses include both malignant and benign diseases that can cause transudate. In particular, for elderly patients for whom further examinations (such as thoracentesis) are risky due to complex underlying diseases or poor health conditions, ultrasound technology, which is harmless and highly specific, may be useful to help clinicians determine whether further examinations for malignant diseases should be performed, thereby helping patients avoid unnecessary radiological or invasive examinations. Due to its easy accessibility and high sensitivity, ultrasound elastography can be used as an initial tool for screening patients with pleural effusion of an unknown aetiology. 
Several limitations exist in our study. First, the sample size of this single-centre study was relatively small, which may have affected the determination of the cut-off elasticity indices for ultrasound elastography. Thus, further exploration should be conducted in a larger population of outpatients, and comparisons of the diagnostic yield of ultrasound elastography should be performed by radiologists and others to simplify diagnostic procedures. As pulmonologists can master techniques such as endobronchial ultrasound-guided transbronchial needle aspiration and rapid on-site evaluation of transbronchial needle aspiration specimens after receiving training [26], we believe that pulmonologists can use ultrasound elastography after training. The use of ultrasound elastography to evaluate the liver is more complicated than its use to evaluate the pleura due to the complex anatomy of the liver, and it has been reported that a 1-year learning curve, or the equivalent of 130 examinations, is needed to master using ultrasound elastography to evaluate the liver [27]. Thus, we believe that the learning curve for examining the pleura by ultrasound elastography would be shorter than that for examining the liver by ultrasound elastography. However, the exact learning curve that would be needed for a pulmonologist to master the use of ultrasound elastography for pleural examination remains to be explored. Second, six cases were excluded from the data analysis due to the lack of a definitive diagnosis, which may have affected the accuracy of our results. Third, we enrolled the entire development set first and then the entire validation set, which may have resulted in the observers gaining experience and skill during development set data acquisition that could have resulted in improved performance during validation set data acquisition, thus leading to bias. Fourth, we did not perform ultrasound elastography-guided pleural biopsy, although this technique better illustrates the value of ultrasound elastography for diagnosing MPE. Thus, a prospective multicentre study with an adequate sample size should be conducted in the future.

\section{Conclusion}

With SWEmean at $47.25 \mathrm{kPa}$, pleural ultrasound elastography may be a better technique than TUS for differentiating MPE from benign pleural disease, especially in countries with a high tuberculosis burden.

Acknowledgements: For their continuous support and assistance with patient screening, we appreciate the help of Wei Wang, Jiang-wei Ma and Zan-feng Wang (Dept of Respiratory and Critical Care Medicine), Xue-jun Hu and Xiao-nan Wang (Dept of Geriatrics), Yun-peng Liu, Ying Chen, Ping Yu, Bo Jin and Ming-fang Zhao (Dept of Medical Oncology), Chun-wei Wu (Dept of Cardiology), Lin-lin Liu and Yi Fan (Dept of Nephrology), Jing Tong (Dept of Gastroenterology) and Bao-cheng Deng (Dept of Infectious Disease) of the First Hospital of China Medical University (Shenyang, China).

Conflict of interest: None declared.

\section{References}

1 Hooper C, Lee YC, Maskell N, et al. Investigation of a unilateral pleural effusion in adults: British Thoracic Society Pleural Disease Guideline 2010. Thorax 2010; 65: Suppl. 2, ii4-ii17.

2 Bibby AC, Dorn P, Psallidas I, et al. ERS/EACTS statement on the management of malignant pleural effusions. Eur Respir J 2018; 52: 1800349.

3 Lin X, Chang C, Wu C, et al. Confirmed value of shear wave elastography for ultrasound characterization of breast masses using a conservative approach in Chinese women: a large-size prospective multicenter trial. Cancer Manag Res 2018; 10: 4447-4458.

4 Rago T, Scutari M, Loiacono V, et al. Low elasticity of thyroid nodules on ultrasound elastography is correlated with malignancy, degree of fibrosis, and high expression of galectin-3 and fibronectin-1. Thyroid 2017; 27: $103-110$.

5 Tian WS, Lin MX, Zhou LY, et al. Maximum value measured by 2-D shear wave elastography helps in differentiating malignancy from benign focal liver lesions. Ultrasound Med Biol 2016; 42: 2156-2166.

6 Marasco G, Colecchia A, Colli A, et al. Role of liver and spleen stiffness in predicting the recurrence of hepatocellular carcinoma after resection. J Hepatol 2019; 70: 440-448.

7 Buderer NM. Statistical methodology: I. Incorporating the prevalence of disease into the sample size calculation for sensitivity and specificity. Acad Emerg Med 1996; 3: 895-900.

8 Diacon AH, Theron J, Bolliger CT. Transthoracic ultrasound for the pulmonologist. Curr Opin Pulm Med 2005; 11: 307-312.

9 Koenig SJ, Narasimhan M, Mayo PH. Thoracic ultrasonography for the pulmonary specialist. Chest 2011; 140: 1332-1341.

10 Görg C, Restrepo I, Schwerk WB. Sonography of malignant pleural effusion. Eur Radiol 1997; 7: 1195-1198.

11 Brandenburg JE, Eby SF, Song P, et al. Ultrasound elastography: the new frontier in direct measurement of muscle stiffness. Arch Phys Med Rehabil 2014; 95: 2207-2219.

12 Lacourpaille L, Hug F, Bouillard K, et al. Supersonic shear imaging provides a reliable measurement of resting muscle shear elastic modulus. Physiol Meas 2012; 33: N19-N28.

13 Azizi G, Keller JM, Mayo ML, et al. Thyroid nodules and shear wave elastography: a new tool in thyroid cancer detection. Ultrasound Med Biol 2015; 41: 2855-2865.

14 Qureshi NR, Rahman NM, Gleeson FV. Thoracic ultrasound in the diagnosis of malignant pleural effusion. Thorax 2009; 64: 139-143.

15 Venekamp LN, Velkeniers B, Noppen M. Does 'idiopathic pleuritis' exist? Natural history of non-specific pleuritis diagnosed after thoracoscopy. Respiration 2005; 72: 74-78. 
16 Davies CW, Gleeson FV, Davies RJ. BTS guidelines for the management of pleural infection. Thorax 2003; 58 : Suppl. 2, ii18-ii28.

17 Light RW, Macgregor MI, Luchsinger PC, et al. Pleural effusions: the diagnostic separation of transudates and exudates. Ann Intern Med 1972; 77: 507-513.

18 Youden WJ. Index for rating diagnostic tests. Cancer 1950; 3: 32-35.

19 Bugalho A, Ferreira D, Dias SS, et al. The diagnostic value of transthoracic ultrasonographic features in predicting malignancy in undiagnosed pleural effusions: a prospective observational study. Respiration 2014; 87: 270-278.

20 Bianchi C, Bianchi T. Malignant mesothelioma: global incidence and relationship with asbestos. Ind Health 2007; 45: 379-387.

21 Robinson BM. Malignant pleural mesothelioma: an epidemiological perspective. Ann Cardiothorac Surg 2012; 1: 491-496.

22 Shaw JA, Irusen EM, Diacon AH, et al. Pleural tuberculosis: a concise clinical review. Clinical Respir J 2018; 12: 1779-1786.

23 Ryan H, Yoo J, Darsini P. Corticosteroids for tuberculous pleurisy. Cochrane Database Syst Rev 2017; 3: CD001876.

24 Malhotra P, Aggarwal AN, Agarwal R, et al. Clinical characteristics and outcomes of empyema thoracis in 117 patients: a comparative analysis of tuberculous vs. non-tuberculous aetiologies. Respir Med 2007; 101: 423-430.

25 Mutsaers SE, Prele CM, Brody AR, et al. Pathogenesis of pleural fibrosis. Respirology 2004; 9: 428-440.

26 Bonifazi M, Sediari M, Ferretti M, et al. The role of the pulmonologist in rapid on-site cytologic evaluation of transbronchial needle aspiration: a prospective study. Chest 2014; 145: 60-65.

27 Fraquelli M, Baccarin A, Casazza G, et al. Liver stiffness measurement reliability and main determinants of point shear-wave elastography in patients with chronic liver disease. Aliment Pharmacol Ther 2016; 44: 356-365. 